J. Radiat. Res., Supplement, 149-164, (1975)

Review of Thirty Years Study of Hiroshima and Nagasaki Atomic Bomb Survivors

III. FUTURE RESEARCH AND HEALTH SURVEILLANCE

\title{
B. Future Research
}

\author{
BEEBE, G. W. and HAMILTON, H. B.* \\ Departments of Epidemiology and Statistics and of Clinical Laboratories, \\ Radiation Effects Research Foundation**, Hiroshima, 730, Japan. (Received April 21, 1975)
}

Much remains to be learned from continued monitoring of the medical experience of the Hiro. shima and Nagasaki A-bomb survivors. Many effects are so small, or have such long latent periods, that there is not even now a complete catalogue of effects visible in this large population. There is as yet no evidence of a mutagenic effect, but biochemical and cytogenetic approaches now offer powerful tools for a final effort to derive information that may set limits on the size of the doubling dose for mutations in man. The list of specific neoplasms induced by the whole-body exposure to the A-bomb radiation remains incomplete, and the quantitative dose-response aspects of established carcinogenic effects remains to be worked out in relation to time, host factors, linear energy transfer (LET) characteristics of radiation, and risk factors other than radiation. The dose-response estimates must be made, where possible, in terms of tissue dose. If there is to be any definitive test of the hypothesis of radiation accelerated aging in man, it will doubtless come from observations on the A-bomb survivors.

The experience of the A-bomb survivors can make no direct contribution to knowledge of dose rate, and is much too small to provide direct information in the region of low dose. Nevertheless, as better knowledge of mechanisms of radiation damage and repair accumulates from laboratory experimental programs, this unique human experience will be of increasing value to radiation biology.

Continued monitoring of the health and medical experience of the A-bomb survivors will depend on the voluntary cooperation of the subjects and their families, and on the interest of the physicians in the community. Present indications are that the research would gain from a closer association with medical care and health maintenance programs.

\section{INTRODUCTION}

Future energy requirements will have to be met in large part by the release of atomic energy. The process of switching from fossil fuels to atomic fuels in electric power plants is already well under way, both in Japan and the U.S. At the same time increasing concern is being expressed on all sides about the human costs associated with the use of atomic energy. Although some of the deleterious effects of ionizing radiation on man have been known for 7 decades or more, and the past 35 years have been a period of greatly intensified research, the definitive knowledge with which to assess risks in terms of health and survival is not at hand; large uncertainties still characterize all present estimates of effects, whether they be genetic or somatic. Much more, and more definite, information is needed before we can accurately assess the human cost of specific exposure levels that may be proposed in the interest of providing electric

* Gilbert W. Beebe, Ph. D. and Howard B. Hamilton, M. D.

* Formerly the Atomic Bomb Casualty Commission

放射線影響研究所，伀島方比治山公園 6-2, 干 730 
power at reasonable cost.

Animal models have been of tremendous importance in clarifying the range of effects of ionizing radiation and the mechanisms by which these effects are produced. Essential though these findings are to the understanding of human effects, they do not translate directly into the human experience in any quantitative sense; only human data are, in the end, entirely satisfactory as a basis for assessing human risk. The only presently usable human experiences are those arising from the pursuit of certain occupations, from medical diagnosis and therapy, and from the two A-bombs of WW II. The last of these is by far the most important, judged both in terms of knowledge already gained and of future potential ${ }^{1-4}$. Nevertheless, the experience of the A-bomb survivors, or of any other conceivable human experience, will not provide any final answer to the question: What is a maximum permissible dose at the population level? This is not only because of the indeterminacy implicit in cost-benefit calculations where costs are measured in human terms, but also because the dose region of interest, and of controversy, is extremely low, well below $1 \mathrm{rem} / \mathrm{year}$, where the risk is too small to be demonstrated empirically, whether in man or mouse, and the statistical requirements as to precision of dose and as to number of subjects cannot be met. At present, in the absence of sufficient theoretical knowledge about radiation injury and repair mechanisms, and of a demonstrated consistency between experimental findings and survey results for man, the human data pertaining to high doses and, generally, to high dose-rates, are being used to estimate low dose effects by means of interpolation. The way in which such interpolation is to be carried out is the subject of active controversy ${ }^{5,6}$.

In the cities of Hiroshima and Nagasaki, where the experience of the A-bomb survivors must be obseryed, the world-wide needs for scientific information on the effects of ionizing radiation, or even its national needs, as Japan increases its use of atomic fuels in generating electric power, are of less immediate interest than are the needs of the survivors themselves. What does a research program have to offer them? Of necessity theirs is a marginal benefit, the greater benefit going to those whose future exposure to ionizing radiation may be reduced by action based on knowledge of the untoward effects of radiation upon the health and survival of the A-bomb victims themselves. But if the effects of A-bomb exposure upon the health of survivors cannot be prevented, it is not too much to ask that the observational process incident to the monitoring of their experience make a positive contribution to their health care, e. g., that it facilitate early diagnosis and treatment. The scientific information itself, specifying risk of disease, death, or genetic defect as a function of dose and host characteristics, is of less certain benefit to the survivor. Since the great majority of survivors received less than 5 rads, and no effect has yet been shown in A-bomb survivors below 20 rads, it should somehow be possible to reassure the great bulk of the survivors with respect to their very low risk of untoward late effects of atomic radiation. Whether, in fact, the research output does or can have such reassurance value deserves serious study.

\section{PRESENT NEEDS}

Several recent authoritative reports, one by the United Nations Scientific Committee on the Effects of Atomic Radiation, ${ }^{4}$, the second by the U.S. National Academy of 
Sciences Committee on the Biological Effects of Ionizing Radiation ${ }^{32}$, and the third by the National Academy of Sciences Committee on Nuclear Science ${ }^{7}$, summarize what is presently known of the effects of ionizing radiation on man. Here, we need only outline that knowledge in a general way, with emphasis on lacunae toward which future research on A-bomb survivors must be directed.

\section{Genetic Effects}

Since the classic experiments of Muller on fruit flies it has been clear that ionizing radiation increases mutation rates, and in the intervening years this has been shown for other species, including mammals, but not man. Since the major genetic effect of interest, gene mutation, is thought to be a linear function of dose, the measure of genetic effect that has come to be the target of estimation procedures is the doubling dose, i. e., the dose that will double naturally occurring mutation rates. Beginning with the NAS BEAR Report in $1956^{1,2)}$ such estimates have ranged from 30 to $80 \mathrm{R}$, with $40 \mathrm{R}$ a likely value, to the 1972 UNSCEAR estimates of $100 \mathrm{R}$ for males, and the $1972 \mathrm{BEIR}$ estimates of 20 to $200 \mathrm{R}$. However, all these estimates are based on extrapolation from experimental data, especially from the mouse. The fact that they are in good general agreement is not itself evidence of their applicability to man, but Abrahamson et al.8) have recently provided evidence of uniformity in radiation induced mutation rates for different species that inspires additional confidence in such extrapolation. Nevertheless, the need is for human data that will, first, attest to the validity of such order-of-magnitude estimates for man and, second, reduce the error of estimate below those obtained from the experimental data.

The largest human population ever studied for the genetic effects of radiation is the population of A-bomb survivors in Hiroshima and Nagasaki. A search for genetic effects made by Neel et al., based on a nearly complete registration of births in both cities over the period 1948-1952 (see also Kato, earlier paper in this series), failed to show any evidence of radiation-induced genetic defects in the offspring $\left(\mathrm{F}_{1}\right)$ of heavily exposed parents, in comparison with children of either lightly exposed or non-exposed (not in the city at the time of the bomb) parents. The study extended to 77,000 conceptions, a size thought sufficient to detect a doubling of congenital malformations, etc. with $90 \%$ probability. Nonetheless, human geneticists assume that radiation effects similar to those seen in other species must also occur in man, and the failure to observe an effect in man can only mean that it was too small to be detected by the methods used and the size of sample available.

With the conclusion of the first genetic study of the A-bomb survivors' children, continued genetic investigation focussed on mortality in $F_{1}$ born between 1946 and 1958 . This study was undertaken to guard against the risk of missing genetic changes leading to later phenotypic expression sufficient in magnitude and frequency to result in observable changes in mortality experience ${ }^{10}$. So far, no significant association has been seen between the death rates of $F_{1}$ children and parental dose. (See Kato, pp 67 to 74, this issue)

Recently there has been a virtual revolution in genetic methodology, so that micro methods can now be used in lieu of the earlier macro methods to search for radiation induced genetic effects. First among these was the development of cytogenetic techniques enabling the geneticist to detect transmitted chromosome defects which might 
result from parental exposure. In an earlier paper in this series Awa raises the possibility that sex chromosome anomalies and structural autosomal anomalies may occur more frequently in the children of irradiated parents than in those of non-exposed parents, though the discrepancies thus far found lie within the range of chance variation $^{11)}$ (also, Awa, this journal). Clearly these studies need to be continued to the point where more definite conclusions can be reached.

A further refinement of the micro approach to the search for genetic effects is the recent emergence of techniques, such as starch gel electrophoresis and high speed centrifugal analysis, that, with relative ease and at low cost, can detect subtle quantitative and qualitative protein variation in human tissues, notably red cells and serum. These biochemical methods, coupled with family studies, could be used to detect 'new' mutations of proteins in the $F_{1}$ for comparison with 'naturally' occurring mutations in each generation. Neel recently proposed that, with these techniques, a survey of the $F_{1}$ for mutant proteins might well serve as the ultimate effort to use the A-bomb survivors' experience for obtaining an estimate of the doubling dose for mutations in $\operatorname{man}^{12}$. A three year pilot study ${ }^{13}$ in Hiroshima and Nagasaki has confirmed the feasibility of his proposal. It has, in addition, yielded considerable data on normal biochemical variation in man, inasmuch as the sampling in this preliminary investigation, as well as family studies of rare variants actually found, has been restricted largely to the parent generation. Inauguration of a full-scale biochemical genetic study in the $F_{1}$ is now under serious consideration.

Somatic Effects

In sharp contradistinction to genetic effects, late somatic effects must by visualized in a variable time-frame which is perhaps best illustrated with reference to leukemia. Major characteristics of interest are:

period of latency-After what period of time following exposure does the effect begin? duration of effect-For what length of time is a non-zero effect observed? How does the effect move over time - the build-up, the peak or plateau, and the subsidence to zero?

Other dimensions of importance for established effects are

infuence of dose rate-Does a divided dose over time have the same effect as one delivered all at once?

linear energy transfer (LET) characteristics of the radiation-Does LET influence the effect?

functional form of dose response-Is the (generally) more conservative linear hypothesis the best basis for interpolating to the region of low dose? For genetic effects it does seem to be; for somatic effects there is very little information for man.

host characteristics-Age and sex are of basic importance, but other characteristics may be of interest for somatic effects, e. g., smoking, endocrine balance, and family history. Until more is known about mechanisms of radiation carcinogenesis, the significance of host factors necessarily remains an open question, and one deserving serious attention.

tissue dose-For somatic effects the organ dose and its distribution within the target tissues provide the best expression of the radiation received.

The experience of the A-bomb survivors can make no direct contribution to knowl- 
edge of dose-rate effect, but can be used indirectly, e. g., in comparisons with other samples exposed to similar doses at low dose rates. The experience is also deficient with respect to tissue dose; at present fetal tissue doses are being calculated at $\mathrm{ABCC}$ on the basis of the work of Hashizume et al. ${ }^{14)}$ and of Jones et $a 1^{15)}$. It is expected that the Oak Ridge National Laboratory dosimetry group will also soon provide the basis for calculating tissue doses of the thyroid, breast and other organs. In the exploration of all other dimensions of the effects of ionizing radiation on man the experience of the A-bomb survivors is the most informative human experience available for study. Of particular interest is the opportunity to contrast the effects of the two bombs, which, because of their different construction ${ }^{16)}$, differed widely in the neutron vs. gamma components of the dose delivered to the victims. Such contrasts may provide important information on the influence of LET upon the effects of any particular radiation dose.

Known and potential somatic effects are of varied importance for health and survival, as follows :

1. Cataract of the lens has been shown to be an effect of radiation for man (Abomb survivors, cyclotron workers, patients who have received radiotherapy) as well as for experimental animals, for both gamma and neutron radiation ${ }^{17,18)}$. The evidence is that cataracts and lesser opacities of the human lens will not follow exposure in the low dose region of interest, say, for radiation workers (less than 5 rems per year), so that there is little interest in further refinement of risk estimates for cataract induction in man on the part of those concerned solely with the hazards of low-dose radiation ${ }^{7}$. Nevertheless from the broader standpoint of radiation biology as a whole it would be desirable to have more precise information on the shape of the dose-response curves for neutrons vs. gamma radiation, on the influence of dose-rate upon dose-response, on the possibility of a long latent period associated with doses in the intermediate range for A-bomb survivors, and on the possibility of progression in later life.

The initial observations on cataracts among A-bomb survivors ${ }^{19}$ ) were made before the present system of dosimetry was introduced ${ }^{20}$, and also before the $A B C C$ fixed cohorts were defined $^{21}$. Nevertheless it should be possible to estimate the dose-response curve for each city. Whether it is possible to estimate the relative biological effectiveness (RBE) for neutrons is much less certain. Further, any younger persons observed to have cataracts in the studies of 20 years ago should be followed to determine whether progression occurs in later life. There is no reason to suppose that new radiation cataracts would continue to appear, or that exposed individuals at the time of the bombing (ATB) would be at greater risk of developing senile cataracts.

2. Growth and development are demonstrably affected by ionizing radiation, for $\operatorname{man}^{22)}$ (see also Blot, pp 82 to 88 , this issue) as well as for experimental animals; the effect is especially clear in man following irradiation of the fetus during the first 18 weeks of pregnancy and extends to mental retardation ${ }^{23}$ as well as to physical size. The effect of irradiation after birth is less firmly established for man, principally because of the possible confounding of dose and early nutritional deprivation among the A-bomb survivors, For man there is no information on the influence of dose-rate, on differences between gamma radiation and neutrons in dose-effectiveness, or on the functional form of the dose-response curve.

All the observations with respect to growth and development that can be made on 
A-bomb survivors exposed in utero, in infancy, and in early childhood, have already been made, but further analysis is indicated, especially of ABCC data on in utero subjects, to clarify (a) the shape of the dose-response curve and (b) any differences between gamma radiation and neutrons as to dose-response. For the in utero subjects tissue doses are now available and appropriate re-analyses are under way.

3. Accelerated aging was the interpretation originally placed on experiments in which it appeared that animals were dying more rapidly from cancer and from all other causes as well ${ }^{24}$. . In recent years this interpretation has come under severe methodologic criticism ${ }^{25}$, 26) and the hypothesis of radiation accelerated aging no longer enjoys the wide support it had 10 to 15 years ago. Data on man have not been generally supportive, and there has been no indication of a nonspecific, accelerated senescence in the mortality experience of the A-bomb survivors. The one report on man that has seemed to provide some support for the hypothesis is the mortality follow-up study of U.S. radiologists in comparison with other medical specialists ${ }^{27}$. Seltser and Sartwell found excess mortality not only from leukemia and from other forms of cancer, but from cardiovascular diseases and from other causes as well. In fact, the excess deaths they calculated for the experience of U.S. radiologists for the years 1935-1958 number 11 for leukemia, 48 for other cancers, and 169 for all other causes. The significance of their findings, of course, rests upon an untestable proposition, namely, that apart from radiation, the expectation of life among U.S. radiologists was the same as that for the ophthalmologists and otolaryngologists with whom they were compared. Court-Brown and Doll, in their smaller study of British radiologists, found only an excess of cancer deaths in comparison with other physicians and men in social class $1^{28}$.

Not only for man but more generally, all radiation-induced life-shortening tends now to be explained in terms of carcinogenesis, but only after another 20 years or more could negative findings as to causes of death other than cancer be taken as conclusive, especially for those irradiated in utero and as infants and children. The experience of the survivors, monitored over a sufficiently long period by such means as the ABCC Life Span Study ${ }^{29}$, will eventually provide the basis for a definitive test of the hypothesis at the level of mortality.

4. Cytogenetic changes in human lymphocytes have been conclusively shown to be radiation induced in $\operatorname{man}^{30}$, and for the $\mathrm{A}$-bomb survivors, at least, preliminary doseresponse curves are available ${ }^{31}$. More information is needed in the low-dose-region with respect to differences between gamma radiation and neutrons in regard to dose response function, however. Perhaps the greater need is for a determination of the biologic significance of such abnormal changes: are they simply markers, or do they have predictive value for future events of clinical significance?

5. Impairment of immune response has been shown for $\operatorname{man}^{4)}$, and there is at least some suggestive evidence for A-bomb survivors ${ }^{32}$. However, information is needed on the different aspects of immune responses, on dose-response, and on the dependence of dose-response, if any, upon the linear-energy transfer (LET) characteristics of the radiation exposure, the dose-rate, host characteristics, and calendar time. There is no human information on the role radiation-induced lowering of immune response may play in radiation carcinogenesis. Whether an impairment of the immune response can be detected in $A$-bomb survivors so long after radiation must first be determined, and 
a preliminary study is presently under way at $\mathrm{ABCC}$ to estimate thymus-derived lymphocyte immune competence, to estimate cell population size (as a possible indicator of $T$-cell depression by radiation), and to search for any relation between chromosomal aberration and immune competence ${ }^{33}$. If an effect can be detected it will then become important to describe the impairment in dose-specific terms, to assess the significance of host factors, especially age ATB, and to examine the influence of LET. There is also interest in auto-antibody levels and in the possible appearance of auto-immune diseases in those who were exposed to radiation at an early age. Such studies could be done as part of the Adult Heath Study as presently conducted at ABCC.

6. Cancer is the chief somatic effect of clinical and public health significance, both leukemia and solid tumors. The tumors thought to be caused by atomic radiation are cliscussed by Beebe and Kato, (pp 97 to 107 this issue). Approximate dose-response functions exist for leukemia, breast cancer, and lung cancer, but these need refinement especially with regard to their dependence upon host factors and LET characteristics of the radiation exposure. Uncertainties as to the cancers of the salivary glands and of the gastro-intestinal tract need to be resolved, and the search for other sites, and for more specific information on cell type, must be continued. The relationships among immune competence, radiation dose, and carcinogenesis especially merit further attention. Among the host factors of particular significance is age at exposure. Present uncertainties as to the human cost of radiation exposure revolve in large part around the effects of low doses to the fetus; there is uncertainty as to the size of these effects, and as to their duration ${ }^{34-39}$ ). An effect persisting throughout the lifetime of the exposed individual could have a very large effect on estimates of the human cost of almost any level of ionizing radiation ${ }^{3}$. The future experience of the A-bomb survivors, properly studied, will provide a tremendous amount of important information on the carcinogenic effect of ionizing radiation. Overall, the leukemogenic effect seems clearly to be wear. ing off, but an excess may continue for some time among those at the older ages ATB. In addition to providing definitive information on the behavior of the excess over time, future research on leukemia should be directed at the more precise estimation of the dose-response function in Nagasaki survivors, and its relation to that for Hiroshima survivors.

With the cooperation of hematologists in Japanese universities, especially those in Hiroshima and Nagasaki, a complete leukemia registry has been maintained for the two cities for many years ${ }^{40}$. Whether the cases in the registry are, in fact, numerous enough to provide a stable estimate of the Nagasaki dose-response curve, and to differentiate it from the Hiroshima dose-response curve, and whether any such differentiation may also involve consideration of type of leukemia and age of exposed, remain to be ascertained. Since the number of A-bomb survivors eligible for the leukemia registry is much larger than the number in the ABCC Life Span Study sample, there is some possibility that, if accurate estimates could be made of the dose-distribution of the population of all survivors resident in the two cities in the period 1950-1974, more could be learned about the difference between cities, and about the shape of the dose-response curve for Nagasaki. Present indications are that future cases occurring within the Life Span Study sample will be too few to improve materially the present means of answering these important questions. It may even be too small to monitor closely the final 
stages of subsidence of the leukemogenic effect. Moreover, although the death certificate has proved highly accurate in the past as to leukemia generally, it will be far less so as to type of leukemia, determination of which will for some time continue to require ascertainment at the level of morbidity.

It is with respect to the solid tumors that so much work remains to be done. But the great potential contribution of the experience of the A-bomb survivors will not be realized if this is attempted merely on the basis of death certificates. Adequate though they have proved to be with respect to the detection of cancer undifferentiated as to type or site, they are inadequate as to most specific sites of cancer, and provide no information on cell type. The ABCC Life Span Study (LSS) sample provides an excellent cohort for studying the future cancer experience as it unfolds but it needs to be supplemented by a much more active autopsy program and by cancer registries that will provide more accurate and more extensive information on cancer. The ABCC autopsy program was highly successful in the period 1961-1966, attaining an overall autopsy rate of $45 \%$ for the LSS. It has become progressively less successful since that time and the autopsy rate currently stands at about $20 \%$. From the scientific point of view strenuous educational efforts are warranted with both the community at large and the medical practitioners of the community to bring them to a fuller realization of the importance of an autopsy program in the cities of Hiroshima and Nagasaki.

Cancer is not a uniformly fatal disease, and even fatal forms of cancer need not be so immediately. There is great advantage, therefore, in being able to study the occurrence of cancer at the level of morbidity. For thyroid cancer it is absolutely imperative. In the past monitoring the A-bomb survivors for cancer incidence has been possible only on an ad hoc, site-specific, basis in Hiroshima, through negotiation with individual institutions and even departments within institutions. Both cities have had tumor registries sponsored by their city medical associations, with the technical operations of data collection and data processing entrusted to $\mathrm{ABCC}$, but the Hiroshima registry especially has been quite incomplete, as not all institutions have entered into the collaborative pattern. In 1973 the Hiroshima Prefectural Medical Association established a tissue registry and succeeded in gaining the cooperation of pathology departments that had not contributed to the city-sponsored registry. Perhaps the combination of the two registries will enable investigators to approach cancer ascertainment at the level of incidence in Hiroshima in the future, but it will still be difficult to incorporate material from the past. In 1974 the Nagasaki City Medical Association also established a tissue registry that should further the development of pathology diagnosis in Nagasaki.

The experience of the A-bomb survivors should prove decisive as to the existence of a general carcinogenic effect vs. a limited number of site-specific effects, within the range of whole-body dose consistent with survival. Thus, skin cancer will probably never appear in the catalogue of cancers attributable to ionizing radiation of the $A$-bomb survivors if it requires a partial body dose that, extended to the whole body, would be incompatible with survival. But, a general carcinogenic effect aside, given adequate surveillance of the A-bomb survivors as a whole, or of the subjects of the ABCC LSS, there is every expectation that a definitive list of tumors induced by whole-body radiation can be developed, and that their dose-response characteristics can be determined. For each tumor the desiderata are: 
size of effect

period of latency

duration of effect

LET characteristics of dose-response

influence of host characteristics

with dose expressed as average tissue dose if this be possible. For no tumor is all this information available at this time, and its provision will require many years of dedicated effort on the part of many investigators and supporting staff.

7. Fertility Ionizing radiation has been shown to affect the fecundity of experimental animals, and of man. Although the data for man, derived from the experience of the A-bomb survivors and of the Marshallese and Japanese victims of test fallout in the Pacific, are imprecise, they suggest that, apart from temporary effects on spermatogenesis, impairment is detectable only after high doses (200 to $400 \mathrm{rems}$ ) to the female. ${ }^{3)}$ For the male, the sterilizing dose is thought to exceed the lethal dose. Recent fertility studies by $\mathrm{ABCC}$ investigators ${ }^{41,42)}$ have slown that it is much too difficult to control the effects of voluntary measures taken to limit fertility for the investigator to be able to detect any late effect of radiation upon fertility. Nevertheless, the fertility of the in utero exposed should be monitored in an effort to learn whether their exposure had quantitatively different effects upon their reproductive potential.

8. Effects not yet known At this point in time, 30 years after exposure, effects that have not yet been seen in the experience of the A-bomb survivors, or of other exposed groups, might be left to a program of mortality surveillance (including autopsy) to discover, but only at some risk. The risk is that the clinical and laboratory work that might elucidate the origins of an effect discovered later, e. g., in those who were under ten ATB, would not have been done. Only an active program of clinical surveillance, with a laboratory component updated in response to advances in medical knowledge, can provide reasonable assurance of uncovering effects that are not now known or suspected.

Psychiatric Effects

Although the atomic bombings represent one of the great cataclysmic events in human history, little research seems to have been attempted in order to draw from the psychological experience of the survivors further knowledge of man's potential for coping with disaster, especially in terms of subsequent life history. Not only is such knowledge of value in understanding human nature itself, but it offers at least some possibility of providing a basis for therapy directed at the relief of anxiety about their future health that is incompatible with present biologic knowledge of the effects of radiation on man. There is a very real need for developing therapeutic approaches that would enable the A-bomb survivor to scale down his anxiety to a level more nearly consistent with his objective risk.

\section{SOME IMPLICATIONS OF FUTURE RESEARCH NEEDS}

The problems that remain, if we are to have a reliable scientific basis for protecting the population against unnecessary risk from ionizing radiation, and to understand the mechanisms by which ionizing radiation produces untoward effects, are both numerous and important. Their solution will probably require even more than the life-time monitoring of the great bulk of the survivors. The A-bomb survivors do not provide an 
important resource for learning further about mechanisms, dose-rate, or the effects of . $\alpha$ and $\beta$ particles. In other areas where knowledge is incomplete they have a great contribution to make, if only because other human experiences are generally smaller, of less certain dosimetry, more difficult to observe, or difficult to interpret because of possible confounding between radiation dose and other risk factors. On all these counts the potential scientific value of the A-bomb survivors' experience is quite high. In addition, the studies of survivors made thus far provide a firm basis for research in the future, especially the fixed cohorts developed at $\mathrm{ABCC}$ for the in utero exposed, ${ }^{43}$ ) those exposed after birth, ${ }^{29}$ ) and the $F_{1}$ of exposed parents, ${ }^{10)}$ and the observational procedures applied to these cohorts. ${ }^{21}$ No presently conceivable human studies, however, should be expected to provide direct measures of risk in the low-dose area, below a few rems. Genetic Effects

Although $F_{1}$ births will continue to occur for many years, especially to exposed males, the annual increment of births has already passed its peak and the mobility of Japanese youth poses problems for the genetic investigator. Experience with the ABCC $F_{1}$ mortality sample, consisting of individuals born in the interval 1946-1958, suggests that the number of such subjects resident in Hiroshima and Nagasaki is still declining. From the Adult Health Study experience with exposed subjects under ten years of age at the time of the bomb (born 1935-1945), ${ }^{43}$ it might be supposed that the percentage available to study in the contacting area would be about $55 \%$ and $70 \%$ for males and females respectively in Nagasaki, and 70 and $75 \%$ in Hiroshima, over the next few years. However, a resumption of genetic studies on a major scale, as proposed by Neel, ${ }^{12}$ will require careful preparation of the community and of the A-bomb survivors generally so as not to add further to the anxiety already experienced by the survivors.

Studies at $\mathrm{ABCC}$ have shown that about $80 \%$ of the $\mathrm{F}_{1}$ will come to a clinic to donate blood for cytogenetic studies, but this level of participation has been obtained with the aid of personal visits to the home on the part of trained medical contacting personnel, and an offer of a blood examination or a complete check-up if desired. Other, more direct, methods of collecting blood might have different results. Of particular importance for any future genetic study is its relationship to any $F_{2}$ surveys done in the two cities by the local authorities responsible for administering the health and welfare program for A-bomb survivors. It would seem important that a scientific genetic study not compete with such surveys for $F_{1}$ participation, but be allied with them in such fashion as to strengthen both. Provision also needs to be made for consultation with the parents regarding rarer variant or mutant proteins and their lack of biological or clinical significance, in order to explain the necessity for family studies. Finally, the relation between a genetic study such as Neel has proposed, and the continuing $F_{1}$ cytogenetic study, ${ }^{11)}$ needs to be considered in the same light as its relation to the $F_{1}$ surveys already begun by the city authorities.

Inasmuch as early genetic studies failed to demonstrate effects in the children of the exposed, geneticists advising the U. S. National Academy of Sciences have argued that only the $F_{1}$ generation merits continued study; the magnitude of any genetic effects in the $F_{2}$ would in all probability be too small to be easily measured, and further, an accessible $F_{2}$ population would be even smaller than the $F_{1}{ }^{45}$ ) Nonetheless, while large-scale, direct observations of the $F_{2}$ on a nation-wide basis would seem to be out 
of the question, an $F_{2}$ mortality study could be performed if based on the Japanese family registration system, should current or proposed $F_{1}$ studies (mortality, cytogenetic, and biochemical) produce direct evidence of detectable radiation effects. Although it lacks the promise of the proposed Biochemical Genetics Survey, the $F_{1}$ mortality survey ${ }^{10}$ ) is inexpensive and provides necessary insurance against the risk of missing at least large effects of some totally unexpected nature. Thusfar the study has provided little or no indication of a genetic effect, and there is active interest in expanding the sample, especially at the high-dose end, to give it more power to detect any effect that may exist. Somatic Effects

Since the data files of $\mathrm{ABCC}$ and competent investigative talent continue to be available in the Radiation Effects Research Foundation that replaces ABCC, as well as in other research facilities, it seems necessary to discuss only effects whose evaluation depends on the collection of new information.

These are:

1. Accelerated aging, for which continued mortality and clinical surveillance is required.

2. Cytogenetic changes, tissue culture studies of which should be continued for perhaps 10 years to provide definitive estimates of the dose-response function and an adequate basis for determining the relationship of these changes to subsequent cancer.

3. Impairment of immune response, on which very little information now exists for A-bomb survivors, especially when viewed as a late effect, and that probably will require some years of effort akin to that for cytogenetic changes.

4. Cancer, for which information at the level of both mortality and incidence is needed at least until those under age ten ATB reach age 50 in 1995.

5. Fertility, for which continued surveillance of the in utero exposed is required. ${ }^{46)}$

6. Major chronic disease other than cancer, for which continued mortality and clinical surveillance is required.

The death certificate information needed for the study of accelerated aging and cancer is of the same kind as that needed for the $F_{1}$ mortality study. The cytogenetic work is presently confined to lymphocytes, but might be extended to other tissues. Affiliation with medical care or health maintenance programs would help to insure participation by members of any study sample, especially if repeat examinations are to be performed in the interest of measuring the reliability of the observational process and to follow the course of abnormalities over time. The correlation of cytogenetic abnormalities with subsequent cancer could be accomplished through a death certificate follow-up, but if any positive association were ever found more precise information would be desired.

The information needed to describe fully the carcinogenic action of ionizing radiation is complex and difficult to obtain. At the level of mortality only autopsy will provide accurate and specific information on tumor site and cell type. For its scientific promise to be fulfilled the autopsy will have to become an integral part of medical care in the two communities, essentially a program carried out by the practicing physicians in cooperation with the pathologists of the community. Since about half of the deaths occur at home, ${ }^{47)}$ it would not suffice to confine the program to deaths occurring in hos- 
pitals. To obtain the active support of the practitioner the autopsy program should minister to his need for information and to the needs of the family, and not merely to the scientific need for radiobiological information.

For some years, at least, the detection of cancer (including leukemia) should be at the level of incidence, as has been the case with leukemia in the past. This is not only because the tumors of interest are not all fatal, or rapidly so, but because even for fatal tumors the lag between incidence and mortality may be such as to delay recognition of an upswing in incidence by as much as 10 years. At $A B C C$ the early indications of both lung cancer and breast cancer were obtained from incidence data. ${ }^{48,49}$ ) Screening techniques being none too efficient, and the frequency of cancer such that very large samples are required for all except a few of the most common sites, it hardly seems feasible at this time to base the ascertainment of cancer on a clinical screening program. ${ }^{43}$ The screening approach, however, especially with a standard examination capable of being varied in response to new research hypotheses and techniques, offers advantages with respect to developing information on pathogenesis and on so-called "risk factors". For example, Knittle's 1959-1962 survey of the Adult Health Study subjects for serum pepsin levels and achlorhydria, ${ }^{50}$ with follow-up through 1969 by Pastore et al. ${ }^{51)}$, showed that low serum pepsin levels were associated with the risk of stomach cancer, and that the risk was highest for those with both low serum pepsin and achlorhydria. Similarly, the relation between such factors as blood pressure, uric acid, smoking, serum cholesterol, exercise, diet pattern, etc. and subsequent cardiovascular disease in Japan ${ }^{52 ~ 54)}$ is a matter of basic and continuing interest. Also a clinical sample offers the only reasonably effective mechanism for accurately assessing exposure to medical $x$-ray on a continuing basis, and analyses based solely on T65 dose estimates may err in ignoring the substantial amounts of medical x-ray experienced by some patients.

The only presently feasible way to ascertain cancer is to tie into medical care programs so as to pick up each newly diagnosed case, and for this purpose the tumor registry is the method of choice. Again, this is the model followed in developing information on leukemia ${ }^{55}$ and the tumor registries established in Hiroshima in 1957, and in Nagasaki in 1958, ${ }^{56}$ ) have now been strengthened by a tissue registry in each city.

It is not at present possible to develop information on dose-response of the requisite quality on the basis of all survivors residing in the city, as neither good dose information nor census counts are available for this changing set of survivors. For immediate practical purposes, therefore, the ABCC cohort for the Life Span Study, with its individual dose estimates, ${ }^{20}$ is the most effective sample available. Nevertheless, in leukemia we already have an example of its inadequacy as a basis for estimating dose response for Nagasaki in comparison with Hiroshima, and it may be that no sample of the survivors is large enough to settle this important issue. There are, however, many cases of leukemia in the leukemia registry who are not members of the Life Span Study sample, and therefore the effort to estimate the dose distribution of the A-bomb survivors resident in the two cities from 1950 to 1974 would be well worthwhile.

For research on diseases other than cancer the great need is for a comparable information system on the health of all survivors, a system to which medical investigators may seek access under conditions carefully specified so as to protect the privacy and 
rights of the individual. A medical examination program cannot provide significantly frequent examinations to permit reliable estimates of disease incidence on a routine basis. Sick people do not come for their routine medical examinations. Others, who do come, will often forget to report a hospitalization experienced a year or more previously, or may be unable to give a sufficiently precise history. Those who die may not have been examined for some time and tend to have especially incomplete intervals. For these reasons a necessary supplement to any ongoing clinical examination program is some provision for a centralized collection, under proper safeguards, of complete information on all hospitalized illness. The information on hospitalization could provide an index by means of which case/control studies could be made on the basis of more detailed information in hospital records, or generated by direct observations of the subjects themselves. If such a system were combined with the A-bomb handbook, ${ }^{*}$ and with a death certificate check, it would provide investigators with a wealth of information descriptive of the health of the community and also suited to a wide variety of special research studies. Its existence would greatly expand the usefulness of the tumor registry in each city, not merely for the study of effects of radiation, but more generally, to provide information on cancer that would be useful to the medical profession in its efforts at early diagnosis and treatment.

\section{THE MEDICAL AND INFORMATION NEEDS OF A-BOMB SURVIVORS}

The research needs can be satisfied, in the main, only through the voluntary cooperation of the subjects and their families. If the only need now and in the future were for information that would be obtainable through an autopsy program, it is extremely doubtful that the families of the exposed would cooperate. If information is to be obtained from the survivors, then their needs and wishes inevitably will set limits on the observations that will be tolerated.

The special status of the A-bomb survivors is already recognized in Japanese law providing medical care and compensation, ${ }^{57}$ in the existence of a special A-bomb hospital in each city, in the presence of clinics for A-bomb survivors in both cities, in the establishment of a special A-bomb medical research institute attached to the national university in each city, and in the political attention given to their demands for medical care and other welfare legislation.

But if the research cannot proceed without the provision of service, there is also the prospect that the research can be the better for its integration with a medical service program, at least at the level of participation rates. There may well be disadvantages to the research interest in such an integration, e. g., if it means too many different clinics and hospitals to permit uniform observations, or difficulties in coordinating service and research requirements for scheduling subjects and patients, for handling medical personnel, and the like. But in the main it would appear that the research would gain from a closer association with medical care and health maintenance programs. How such integration might be effected deserves careful thought and discussion on the part of those responsible for medical service in the community.

\section{SUMMARY}

1. To satisfy the need for scientific information obtainable from the experience

\footnotetext{
* a unique, permanent handbook number would seem to be necessary.
} 
of the A-bomb survivors, a formal research program should be continued for another 20 years.

2. The genetics program ( $F_{1}$ cytogenetics and $F_{1}$ mortality) should be augmented to include the proposed study of mutations at the protein level.

3. The somatic effects of major interest are various forms of cancer, but a mortality follow-up should be continued indefinitely to guard against missing other possible major effects, and more serious attention should be given to studies of immune competence.

4. The main tools for continuing cancer research will be the death certificate, the autopsy, and the tumor registry.

5. To be scientifically most effective, and more useful to the subjects themselves, clinical surveillance should be performed in association with the hospitals and clinics of the community responsible for medical care and health maintenance.

6. A program of clinical surveillance, in combination with studies of mortality and cancer, and supplemented by a strong autopsy program, would provide a strong base for studies in the natural history of disease and for the continuing investigation of changes in the spectrum of disease in Japan and of the factors associated with it.

7. Serious consideration should be given to the organization of an information system on the health of all A-bomb survivors, at least at the level of hospitalized illness.

\section{REFERENCES}

1. National Academy of Sciences-National Research Council (1956) The biological effects of atomic radiation. Washington, D. C., NAS-NRC.

2. National Academy of Sciences-National Research Council (1956) Pathologic effects of atomic radiation. Publication 452, Washington, D. C., NAS-NRC.

3. National Academy of Sciences-National Research Council (1972) The effects on populations of exposure to low levels of ionizing radiation (The BEIR Report). Washington, D. C., NASNRC.

4. United Nations (1972) Report of United Nations Scientific Committee on the Effects of Atomic Radiation. Ionizing radiation: Levels and effects. Volume 2. Effects. New York, United Nations.

5. Rossi, H. H. and Kellerer, A.M. (1974) The validity of risk estimates of leukemia incidence based on Japanese data. Radiat. Res. 58: 131-140.

6. Mays, C. W., Lloyd, R. D. and Marshall, J. H. (1973) Malignancy risk of humans from total body $\gamma$-ray irradiation. Proceedings of Third International Congress. Int. Radiat. Prot. Assoc., Washington, D. C.

7. National Academy of Sciences-National Research Council (1974) Research needs for estimating the biological hazards of low doses of ionizing radiations. Washington, D. C., NASNRC.

8. Abrahamson, S., Bender, M. A., Conger, A. D. and Wolff, S. (1973) Uniformity of radiationinduced mutation rates among different species. Nature 245: 460-462.

9. Neel, J. V. and Schull, W. J. (1956) The effect of exposure to the atomic bombs on preg. nancy termination in Hiroshima and Nagasaki. Publication 461, Washington, D. C., National Academy of Sciences-National Research Council.

10. Neel, J. V., Kato, H. and Schull, W. J. (1974) Mortality in the children of atomic bomb survivors and controls. Genetics $76: 311-326$.

11. Awa, A. A., Neriishi, S., Honda, T., Sofuni, T. and Hamilton, H. B. (1973) A cytogenetic survey of the offspring of A-bomb survivors in Hiroshima and Nagasaki, Japan. The 4th International Conference on Birth Defects. 2-8 Sept., 1973, Vienna, Austria. Excerpta Medica No. 297.

12. Neel, J. V. (1970) A program to detect biochemical mutations in Hiroshimn and Nagasaki. 
Memorandum to Dr. Charles Dunham, Chairman, Division of Medicine, National Research Council, Washington, D. C., March 1970.

13. Awa, A. A., Hamilton, H. B., Kato, H. and Neel, J.V. (1974) Studies of the potential genetic effects of atomic radiation. ABCC Research Protocol 5-74.

14. Hashizume T, Maruyama, T., Nishizawa, K. and Nishimura, A. (1973) Dose estirnation of human fetus exposed in utero to radiations from atomic bombs in Hiroshima and Nagasaki. J. Radiat, Res, 14 : 346-362.

15. Jones, T. D., Auxier, J. A., Cheka, J. S. and Kerr, G. D. (1975) In-vivo dose estimates for A-bomb survivors shielded by typical Japanese houses. Health Phys. 28: 367-381.

16. Auxier, J. A., Cheka, J.S., Haywood, F.F., Jones, T.D. and Thorngate, J. H. (1966) Freefield radiation-dose distributions from the Hiroshima and Nagasaki bombings. Health Phys. $12: 425-429$.

17. International Commission on Radiological Protection (1969) Radiosensitivity and spatial distribution of dose. Publication 14, Oxford, Pergamon Press. pp 15-21.

18. Langham, W. H., editor (1967) Radiobiological factors in manned space flight. Publication 1487, Washington, D. C., NAS-NRC. pp 135-144, 199-200.

19. Miller, R. J., Fujino, T. and Nefzger, M. D. (1967) Lens findings in atomic bomb survivors: A review of major ophthalmic surveys at the Atomic Bomb Casualty Commission, 1949-62. Arch. Ophthalmol. 78: 697-704.

20. Milton, R.C. and Shohoji, T. (1968) Tentative 1965 radiation dose (T65D) estimation for atomic bomb survivors, Hiroshima and Nagasaki. ABCC Technical Report 1-68.

21. Beebe, G. W. and Usagawa, M. (1968) The Major ABCC samples. ABCC Technical Report 1268.

22. Wood, J. W., Keehn, R. J., Kawamoto, S. and Johnson, K. G. (1967) The growth and development of children exposed in utero to the atomic bombs in Hiroshima and Nagasaki. Am. J. Pub. Health 57 : 1374-1380.

23. Blot, W. J. and Miller, R. W. (1973) Mental retardation following in utero exposure to the atomic bombs of Hiroshima and Nagasaki. Radiology 106: 617-619.

24. National Academy of Sciences-National Research Council (1960) Biological effects of atomic radiation. Washington, D. C., NAS-NRC.

25. Hoel, D. G. and Walburg, H. E. (1972) Statistical analysis of survival experiments. J. Natl. Cancer Inst. 49: 361-372.

26. Walburg, H. E. Jr. (1975) Radiation-induced life shortening and premature aging. In Advances in Radiat. Biol. ed by Lett, J. T. and Adler, H. Volume 5. New York, Academic Press. pp 145-179.

27. Seltser, R. and Sartwell, P. E. (1965) The influence of occupational exposure to radiation on the mortality of American radiologists and other medical specialists. Am. J. Epidemiol. $81: 2-22$.

28. Court-Brown, W. M. and Doll, R. (1958) Expectation of life and mortality from cancer among British radiologists. Br. Med. J. 2: 181-187.

29. Jablon, S. and Kato, H. (1972) Studies of the mortality of A-bomb survivors. 5. Radiation dose and mortality, 1950-1970. Radiat. Res. 50:649-698.

30. United Nations (1969) Report of the United Nations Scientific Committee on the Effects of Atomic Radiation, Official Records of the General Assembly, 24 th Session, Supp. No, 13 (A/7613). New York, United Nations. pp 98-155.

31. Awa, A. A., Neriishi, S., Honda, T., Yoshida, M. C., Sofuni, T. and Matsui, T. (1971) Chromosome-aberration frequency in cultured blood-cells in relation to radiation dose of A-bomb survivors. Lancet 2: 905-906.

32. Kanemitsu, M., Morita, K., Finch, S. C., Kato, H. and Onishi, S. (1966) Serologic response of atomic bomb survivors following Asian influenza vaccination. Jpn. J. Med. Sci. Biol. 19: 73-84.

33. Akiyama, M., Dock, D. S., Hamilton, H. B., Awa, A. A., Kato, H. and Yamakido, M. (1974) Clinical studies of lymphocyte populations in atomic bomb survivors. ABCC Research Protocol 2-74.

34. Stewart, A., Webb, J. and Hewitt, D. (1958) A survey of childhood malignancies. Br. Med. 


\section{J. $1: 1495-1508$.}

35. Stewart, A. and Kneale, G. W. (1970) Radiation dose effects in relation to obstetric x-ray and childhood cancers. Lancet 1 : 1185.1188.

36. MacMahon, B. (1962) Prenatal x-ray exposure and childhood cancer. J. Natl. Cancer Inst. 28: $1173-1191$.

37. Jablon, S. and Kato, H. (1970) Childhood cancer in relation to prenatal exposure to atomicbomb radiation. Lancet 2: 1000-1003,

38. Diamond, E. L., Schmerle, H. and Lilienfeld, A. M. (1973) The relationship of intra-uterine radiation to subsequent mortality and development of leukemia in children. A prospective study. Am. J. Epidemiol. $97: 283-313$.

39. Oppenheim, B. E., Oriem, M. L., and Meier, P. (1975) The effects of diagnostic x-ray on the human fetus; an examination of the evidence. Radiology 114: 529-534.

40. Ishimaru, T., Hoshino, T., Ichimaru, M., Okada, H., Tomiyasu, T., Tsuchimoto, T. and Yamamoto, T. (1971) Leukemia in atomic bomb survivors Hiroshima and Nagasaki, 1 Oct. 1950-30 Sept. 1966. Radiat. Res. 45: 216-233.

41. Seigel, D. G. (1966) Frequency of live births among survivors of Hiroshima and Nagasaki atomic bombs. Radiat. Res, 28: 278288.

42. Blot, W. J. and Sawada, H. (1972) Fertility among female survivors of the atomic bombs of Hiroshima and Nagasaki. Am. J. Hum. Genet. $24: 613-622$.

43. Kato, H. and Keehn, R. J. (1968) Mortality of in utero exposed children for 18 years after birth, 1945-63. Nagasaki Igakhai Zasshi-Nagasaki Med. J. 43: 829831.

44. Belsky, J. L., Tachikawa, K. and Jablon, S. (1973) The health of atomic bomb survivors: A decade of examinations in a fixed population. Yale J. Biol. Med. 46: 284-296.

45. Crow, J.F. (1964) The Crow subcommittee's recommendation. Letter to Dr. R. K. Cannon of National Academy of Sciences, dated 5 May 1964.

46. Blot, W. J., Shimizu, Y., Kato, H. and Miller, R. W. (1975) Frequency of marriage and live birth among survivors prenatally exposed to the atomic bomb. Am. J. Epidemiol. (in press)

47. Steer, A., Moriyama, I. M. and Shimizu, K. (1973) ABCC-JNIH Pathology Studies, Report 3, Hiroshima and Nagasaki, The autopsy program and the life span study. ABCC Technical Report 16-73.

48. Wanebo, C. K., Johnson, K. G., Sato, K. and Thorslund, T. W. (1968) Lung cancer following atomic radiation. Am. Rev, Respirat. Dis. 98: 778-787.

49. Wanebo, C. K., Johnson, K. G., Sato, K. and Thorslund, T. W. (1968) Breast cancer after exposure to the atomic bombings of Hiroshima and Nagasaki. N. Engl. J. Med. 279:667-671.

50. Knittle, J. L. (1930) Serum pepsin levels in the ABCC-JNIH Adult Health Study sample, Hiroshima. Relation to radiation, ABO blood groups and gastrointestinal disease. ABCC Technical Report 12-60.

51. Pastore, J. O., Kato, H. and Belsky, J. L. (1972) Serum pepsin and tubeless gastric analysis as predictors of stomach cancer: A 10 year follow-up study, Hiroshima. N. Engl. J. Med. 286: 279284.

52. Johnson, K. G., Yano, K. and Kato, H. (1967) Cerebral vascular disease in Hiroshima, Japan. J. Chron. Dis. 20: 545-559.

53. Johnson, K. G., Yano, K. and Kato, H. (1968) Coronary heart disease in Hiroshima, Japan ; A report of a 6-year period of surveillance, 1958-1964. Am. J. Public Health 58: 1355-1367.

54. Kagan, A., Harris, B. R., Winkelstein, W. Jr., Johnson, K. G., Kato, H., Syme, S. L., Rhoads, G. G., Gay, M. L., Nichaman, M. Z., Hamilton, H. B. and Tillotson, J. (1972) Epidemiologic studies of coronary heart disease and stroke in Japanese men living in Japan, Hawaii, and California. Demographic, physical, dietary, and biochemical characteristics. ABCC Technical Report $16-72$.

55. Belsky, J. L., Ishimaru, T., Ichimaru, M., Steer, A. and Uchino, H. (1972) Operations manual for the detection of leukemia and related disorders, Hiroshima and Nagasaki. ABCC Manual 1.72.

56. Ishida, M. (1961) Statistical aspects of tumor registry Hiroshima and Nagasaki. Bull. Int. Stat. Inst. 38 no. 2: 223-234.

57. Atomic Bomb Survivors Medical Treatment Law, 1957 with amendments up to 1968. 


\section{FOREWORD}

I am very pleased to write the Foreword for this Supplement to the Journal of Radiation Research, especially since I have long been concerned with the care of atomic bomb survivors, from my early experience as a radiologist in Hiroshima shortly after the bomb in August, 1945, and attending such patients for months thereafter.

This supplement summarizes our present knowledge of radiation effects on humans. It tells us how difficulties in dosimetry have been overcome and how various symptoms and signs of radiation sickness were recognized and classified. The studies of late somatic effects of radiation, with their dose-response relationships, have been brought up-todate. This is due to careful and painstaking studies carried out during the last 30 years, by many American and Japanese scientists, particularly of the Atomic Bomb Casualty Commission, now the Radiation Effects Research Foundation, in Hiroshima and Nagasaki. Continued survey may prove valuable in clarifying genetic effects among human populations.

Since the peaceful use of atomic energy appears essential for future human existence, we must now provide estimates of the risks from low dose and low dose-rate radiations. The largest body of human data is derived from the studies of the Hiroshima and Nagasaki populations. Additional low dose data, but not low dose-rate rate data may be developed in the future, but will be difficult to obtain.

With this supplement, I see a milestone laid down over the long road to understanding the many different aspects of human radiobiology. This is due to the special efforts of Drs. S. Okada, H. B. Hamilton, N. Egami, S. Okajima, W. J. Russell, K. Takeshita, and G. W. Beebe.

Keisuke MISONO, M. D.

Director, National Institute of Radiological Sciences

Member of Japanese Atomic Energy Commission

Former President of The Japan Radiation Research Society 